\title{
Visual Analysis of Dynamic Networks using Change Centrality
}

\author{
Paolo Federico*, Jürgen Pfeffer ${ }^{\dagger}$, Wolfgang Aigner*, Silvia Miksch* and Lukas Zenk ${ }^{\ddagger}$ \\ *Vienna University of Technology, Austria - \{federico, aigner, miksch $\} @$ cvast.tuwien.ac.at \\ ${ }^{\dagger}$ Carnegie Mellon University, PA, United States - jpfeffer@cs.cmu.com

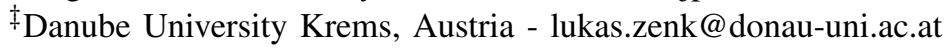

\begin{abstract}
The visualization and analysis of dynamic social networks are challenging problems, demanding the simultaneous consideration of relational and temporal aspects. In order to follow the evolution of a network over time, we need to detect not only which nodes and which links change and when these changes occur, but also the impact they have on their neighbourhood and on the overall relational structure. Aiming to enhance the perception of structural changes at both the micro and the macro level, we introduce the change centrality metric. This novel metric, as well as a set of further metrics we derive from it, enable the pairwise comparison of subsequent states of an evolving network in a discrete-time domain. Demonstrating their exploitation to enrich visualizations, we show how these change metrics support the visual analysis of network dynamics.
\end{abstract}

\section{INTRODUCTION}

The visual analysis of dynamic social networks is an emerging research topic. While several methods, both computational and visual, have been proposed for, and applied to, static networks, there is still the lack of a well-established set of methods supporting the analysis of the evolution of a network.

Simple tasks for analysing the network evolution are the examination and comparison of single occurrences of nodes or links over time, as well as the identification of birth, death and replacement events. Complex tasks require the analysis of compound features, like the shape of change or the rate of change not only at the node level, but also at the group and network level [1].

If complex tasks are challenging, because they demand the simultaneous consideration of temporal and relational aspects, simple tasks might also be hard to solve, even when dealing with relatively small networks. While the visualization of animated graphs has been proposed as an effective technique to support the visual analysis of dynamic networks [2], its real effectiveness has been disputed. In certain cases, for example, static depictions have been shown to be most effective in visualizing trends [3]. In both cases of animation and static visualization, the perceptual phenomenon known as change blindness might occur [4], hampering the detection of changes without specific visual cues. But which types of visual cues are more suited for dynamic networks, and which level of detail or abstraction should they have? One possibility is to highlight all single changes of nodes and links between two subsequent time steps [5]; another one is to compare some structural metrics and visualize their trend over time [6]. The former option points out each and every single change, with the disadvantage that, for large and dense networks, they might be too many and difficult to understand from a global perspective; the latter only provides aggregated structural metrics, that lack local details and might hide certain changes. For example, if we only look at the variation of the degree centrality of a certain node, we might not see any change of its relationships if the number of added links is equal to the number of deleted ones; similarly, if we look at the variation of the betweenness centrality or the closeness centrality we might not see any change if they are symmetric. Conversely, the highlighting of an appearing or disappearing link between two nodes might not help the analyst to understand the impact this change has on the connectivity of the overall structure.

In order to properly support the visual analysis of dynamic networks, enhancing the perception of changes with an appropriate balance of detail and abstraction or, in other words, combining micro and macro features, we introduce the change centrality metric. Also an adequate dynamic layout for node-link diagrams can enhance the perception of change [7]. But while showing relevant changes, it has to minimize unnecessary changes, in order to support the preservation of the user's mental map [8]. As a secondary contribution of this paper, we show how the change centrality can be exploited to optimize a dynamic layout.

\section{RELATED WORK}

While several static metrics have been defined and are widely used for Social Network Analysis (SNA) [9] and Data Mining [10], dynamic metrics are a relatively new research field. In recent years several extensions of existing static metrics to the dynamic case have been proposed as well as new dynamic ones. The time-scale degree centrality has been defined as an extension of the static degree centrality that takes into account both the presence and duration of links [11]. Lerman et al. [12] introduce an attenuation factor to the link duration and on this basis define a centrality metric for dynamic networks. By modelling social interactions as temporal events and taking into account when they occur, Berger-Wolf and Saia [13] introduce a framework consisting of several metrics for the analysis of dynamic social networks. Other approaches consider the time series of static centrality metrics measured over subsequent time windows (e.g. daily or monthly), and compute basic statistics [14], [15]. In general, new metrics have been proposed for multidimensional networks, in which 
the attributes of nodes and links chosen as dimensions are not necessarily referring to time, but for example to the type of relation in multi-relational networks. Berligerio et al. [16] extend the degree centrality from the monodimensional to the multidimensional case and introduce new metrics, namely dimension relevance, dimension connectivity and dimension correlation. Brodka et al. [17] define a cross-layer degree, that is an aggregated node centrality measure across the relations of a multi-relational network.

Most of the above mentioned attempts to combine relational and temporal aspects have the same shortcoming: these two aspects are considered sequentially during the computation, and not simultaneously. Either a relational feature (e.g. degree) is computed for each time point, and then analysed over time (summed, averaged, compared), or a temporal feature is considered (duration, attenuation), and then used to compute a structural metric. Conversely, we aim for a dynamic metric that compares two subsequent time steps taking into account both change events and their relational consequences.

\section{COMPUTING CHANGES IN DYNAMIC NETWORKS}

To address the limitations of existing approaches discussed above, we propose a novel metric for dynamic networks, discuss its properties and meaning, and provide the algorithm for its computation. Furthermore, we introduce a set of derived metrics and show their application to a small exemplary case.

\section{A. Change centrality}

Given a discrete-time dynamic network $\mathcal{G}=G(V, E, T)$, a node $i \in V$ and two time points $t_{1}$ and $t_{2}$, we define the 1-step change ratio:

$$
r_{t_{1}, t_{2}}(i)=\frac{\left|N_{t_{1}}(i) \Delta N_{t_{2}}(i)\right|}{\left|N_{t_{1}}(i) \cup N_{t_{2}}(i)\right|}
$$

where $N_{t}(i)=\left\{j \in V: d_{t}(i, j)=1\right\}$ is the set of nodes connected to node $i$ in one step at time $t$, where $d_{t}(i, j)$ is the geodesic distance between node $i$ and node $j$ at time $t$. Thus, the 1-step change ratio of node $i$ at time $t_{1}, t_{2}$ is defined as the ratio between the cardinality of the symmetric difference of the sets of 1-step neighbours of node $i$ at $t_{1}$ and $t_{2}$ and their union. It can also be seen as the ratio between the number of links added and removed and the number of links added, removed, and remained:

$$
r_{t_{1}, t_{2}}(i)=\frac{\text { added }+ \text { removed }}{\text { added }+ \text { removed }+ \text { remained }}
$$

Considering the example network of Figure 1, node B keeps its links to A and C, but looses its link with D; it looses one of its three connections, then its 1 -step change ratio is $\frac{1}{3}$. Node $\mathrm{D}$ and $\mathrm{E}$ loose all their connections, then their values of 1-step change ratio are both 1 . Nodes $\mathrm{A}$ and $\mathrm{C}$ do not encounter any change, then their values are both zero (see also Table I).

It is worth observing that the 1-step change ratio is the complement to 1 of the Jaccard similarity index $(J)$ [18], defined as the ratio between the intersection and the union:

$$
r_{t_{1}, t_{2}}(i)=1-J=1-\frac{\left|N_{t_{1}}(i) \cap N_{t_{2}}(i)\right|}{\left|N_{t_{1}}(i) \cup N_{t_{2}}(i)\right|}
$$

We name it change ratio because its value is minimum and equal to zero when there is no change in the 1-step neighbours of the node $i$ from $t_{1}$ to $t_{2}$, and it is maximum and equal to 1 when all the neighbours change. Additionally it is symmetric:

$$
r_{t_{1}, t_{2}}(i)=r_{t_{2}, t_{1}}(i)
$$

Now, let us generalize it considering the neighbours reachable in $n$ steps. Given a node $i \in V$ and two time points $t_{1}$ and $t_{2}$, we define the n-step change ratio of $i$ between $t_{1}$ and $t_{2}$ :

$$
r_{t_{1}, t_{2}}^{n}(i)=\frac{\left|N_{t_{1}}^{n}(i) \Delta N_{t_{2}}^{n}(i)\right|}{\left|N_{t_{1}}^{n}(i) \cup N_{t_{2}}^{n}(i)\right|}
$$

where $N_{t}^{n}(i)=\left\{j \in V: d_{t}(i, j)=n\right\}$ is the set of nodes connected to node $i$ in $n$ steps at time $t$. In particular, for $n=0$, by noting that $d_{t}(i, i)=0$, we obtain $N_{t}^{0}(i)=i$.

Finally, we define the change centrality (CC) of node $i$ between time points $t_{1}$ and $t_{2}$ as a linear combination of all the n-step change ratios:

$$
C C_{t_{1}, t_{2}}(i)=\sum_{n=0}^{e_{i}} a_{n} r_{t_{1}, t_{2}^{n}}(i)
$$

where $e_{i}=\max _{t \in\left\{t_{1}, t_{2}\right\}} e_{t}(i)$ is the maximum eccentricity of node $i$ and $a_{n}$ are linear coefficients.

The change centrality of a node is a measure of the change of its connections over time, taking into account its adjacent nodes, the adjacent nodes of the latter and so on. The weight of changes of near and far neighbours depends on the coefficients of the linear combination. By choosing coefficients that decrease with $n$, the changes of farther neighbours will contribute less to the total measure. In particular, if we put $a_{n}=\left(\frac{1}{2}\right)^{n+1}$, because of the convergence of the sum of the geometric series we will obtain a rational-valued, nonnegative, normalized metric:

$$
C C_{t_{1}, t_{2}}(i)=\sum_{n=0}^{e_{i}} \frac{1}{2^{n+1}} r_{t_{1}, t_{2}}^{n}(i)=\frac{1}{2} \sum_{n=0}^{e_{i}} \frac{1}{2^{n}} r_{t_{1}, t_{2}}^{n}(i) \leq 1
$$

Thus, the change centrality of a given node will be equal to zero if no changes have occurred in the connected component that node belongs to. It will have a value greater or equal to 0.5 if the node is present in only one of the two time steps considered. It will get closer to 1 if the latter is true and the network diameter gets larger.

Considering the network of Figure 1 and the corresponding values of the change centrality (Table I), we observe that the nodes with larger values of change centrality are D (which is present in only one of the two time steps) and $\mathrm{E}$ (which looses all of its connections). B has an intermediate value, since it looses one out of its three 1-step neighbours. A and $\mathrm{C}$ are the nodes with the lowest change centrality, because they have changes only in their farther neighbours.

The change centrality is a centrality metric in the sense that it measures how central a node is with respect to the network changes, taking into account whether the node itself has changed (appeared/disappeared), whether its neighbours 

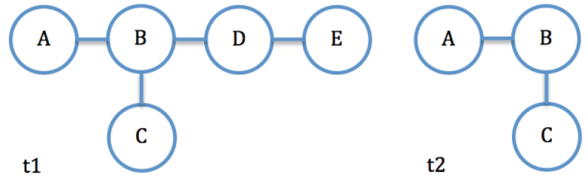

Fig. 1. Example of a dynamic network at two subsequent time steps $(t 1, t 2)$.

TABLE I

VALUES OF CHANGE METRICS FOR THE DYNAMIC NETWORK OF FIG. 1

\begin{tabular}{|ll|}
\hline 1-step change ratio & \\
$r_{t_{1}, t_{2}}(A)=0\left(=\frac{|\emptyset|}{|B|}\right)$ & $r_{t_{1}, t_{2}}(D)=1\left(=\frac{|B+E|}{|B+E|}\right)$ \\
$r_{t_{1}, t_{2}}(B)=0 . \overline{3}\left(=\frac{|D|}{|A+C+D|}\right)$ & $r_{t_{1}, t_{2}}(E)=1\left(=\frac{|D|}{|D|}\right)$ \\
$r_{t_{1}, t_{2}}(C)=0\left(=\frac{|\emptyset|}{|B|}\right)$ & \\
Change centrality & \\
$C C_{t_{1}, t_{2}}(A)=0.125\left(=\frac{1}{2} \frac{|\emptyset|}{|A|}+\frac{1}{4} \frac{|\emptyset|}{|B|}+\frac{1}{8} \frac{|D|}{|C+D|}+\frac{1}{16} \frac{|E|}{|E|}\right)$ \\
$C C_{t_{1}, t_{2}}(B)=0.208 \overline{3}\left(=\frac{1}{2} \frac{|\emptyset|}{|B|}+\frac{1}{4} \frac{|D|}{|A+C+D|}+\frac{1}{8} \frac{|E|}{|E|}\right)$ \\
$C C_{t_{1}, t_{2}}(C)=0.125($ like $\mathrm{A})$ & \\
$C C_{t_{1}, t_{2}}(D)=0.875\left(=\frac{1}{2} \frac{|D|}{|D|}+\frac{1}{4} \frac{|B+E|}{|B+E|}+\frac{1}{8} \frac{|A+C|}{|A+C|}\right)$ \\
$C C_{t_{1}, t_{2}}(E)=0.4375\left(=\frac{1}{2} \frac{|\emptyset|}{|E|}+\frac{1}{4} \frac{|D|}{|D|}+\frac{1}{8} \frac{|B|}{|B|}+\frac{1}{16} \frac{|A+C|}{|A+C|}\right)$ \\
Change eccentricity & Change radius \\
$E_{t_{1}, t_{2}}(A)=2$ & $R_{t_{1}, t_{2}}=0$ \\
$E_{t_{1}, t_{2}}(B)=1$ & Change diameter \\
$E_{t_{1}, t_{2}}(C)=2$ & $D t_{t_{1}, t_{2}}=2$ \\
$E_{t_{1}, t_{2}}(D)=0$ & Stable center \\
$E_{t_{1}, t_{2}}(E)=1$ & $S_{t_{1}, t_{2}}=\{A, C\}$ \\
& \\
\hline
\end{tabular}

have changed, and the ratio between stable and changing neighbours. Moreover, it reflects increasing and decreasing connections, but also zero-balance replacements, that might have little effect on the overall network structure, but are important when analysing ego networks. Furthermore, it gives a measure of how local changes affect far nodes. Taking into account these aspects, the change centrality is a nodelevel metric that combines local and global features, from a relational as well as from a dynamic perspective.

\section{B. Change eccentricity, radius, and diameter}

Considering the definitions of the previous section, we derive additional measures of change at both the node and the network level. Given a node $i \in V$ and two time points $t_{1}$ and $t_{2}$ we define the change eccentricity of $i$ between $t_{1}$ and $t_{2}$ :

$$
E_{t_{1}, t_{2}}(i)=\min \left\{n: r_{t_{1}, t_{2}}^{n}(i) \neq 0\right\}
$$

that can be seen as the geodesic distance between node $i$ and the nearest occurring change. Its value is equal to zero if the node itself has changed (appeared/disappeared), and increases as $i$ gets farther from changes. We put it equal to -1 (to represent infinite distance) if no change has occurred in the set of nodes reachable from $i$. Analogously to the classic node eccentricity, we define two integer-valued network-level metrics on the basis of the change eccentricity, change radius and change diameter:

$$
R_{t_{1}, t_{2}}=\min _{i} E_{t_{1}, t_{2}}(i) \quad D_{t_{1}, t_{2}}=\max _{i} E_{t_{1}, t_{2}}(i)
$$

The change radius is equal to zero if at least one node has appeared or disappeared, it is equal to 1 if only links have changed. The change diameter is a measure of the localization of change within the network: the more locally concentrate the change is, the larger the change diameter is. Both, the change radius and the change diameter are set to -1 if no change has occurred within a connected network.

We can also define a stable center for each time step, as the set of all the nodes that have a change eccentricity equal to the change diameter:

$$
S_{t_{1}, t_{2}}=\left\{i \in V: E_{t_{1}, t_{2}}(i)=D_{t_{1}, t_{2}}\right\}
$$

In Table I we show the values of change eccentricity, change diameter, change radius, and stable center for the simple dynamic network of Figure 1. Node D is the node where the change is localized: its change eccentricity, i.e. its distance from the change, is zero, that is also the change radius of the network. Nodes B and E are close to the disappearing node, so their change eccentricity is equal to 1 . Nodes $\mathrm{A}$ and $\mathrm{C}$ are 2 steps far from the change, so the value for both is equal to 2 . As this is the maximum value, it is also the change diameter and identifies these nodes as the stable center of the network.

\section{Algorithm}

The change centrality (CC) for all the nodes of a graph $G$ between $t 1$ and $t 2$ can be computed by using the following algorithm:

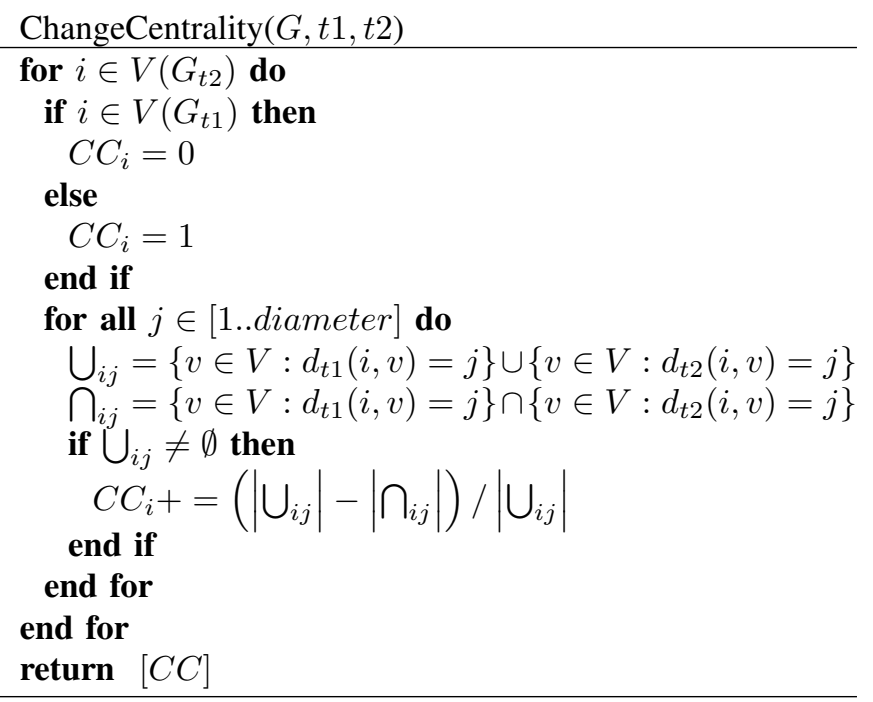

\section{VISUALIZING CHANGES IN DYNAMIC NETWORKS}

In this section we discuss how the metrics we have introduced above can be visually exploited for identifying changes, comparing subsequent time steps and detecting temporal trends; in other words, how these metrics can support the visual analysis of dynamic networks.

We will use a research prototype software, ViENA [19], based on a visual analytics paradigm. Visual Analytics can be seen as an integrated approach combining visualization, human perception and cognition, and data analysis [20]. According to this paradigm, ViENA combines interactive visualizations with a computational kernel, that provides several static and dynamic SNA metrics as well as the change measures we 
introduce in this paper. As for the visualization, we refer to three views: in the juxtaposition view, node-link diagrams of different time points are placed side by side (Fig. 2); in the $2.5 \mathrm{D}$ view, the diagrams are drawn upon transparent parallel planes, stacked along the horizontal axis (Fig. 3); in the superimposition the diagrams are overlaid, links are omitted but node trajectories are emphasized using arrows and transparency (Fig. 4).

The presented change measures can enrich the visualization and supporting visual reasoning and analysis. In detail, we show how they can be: (1) mapped to node size and colour, to facilitate the identification of changes and the comparison of network structures over time; (2) taken into account for the computation of an optimized dynamic layout.

The dataset we use is a real-world dynamic multi-relational network consisting of 38 employees of a small organization and their professional relationships (e.g., acquaintance, communication, advice, collaboration), collected by 4 surveys during 14 months [21]. Since the analysis of multi-relational networks is out of scope of this work, we only refer to and visualize mono-relational networks extracted from this multirelational dataset.

\section{A. Node size and colour}

A typical way to enrich a node-link diagram by using computed node attributes is mapping them to the sizes and the colours of nodes. Figure 2 is a juxtaposition view of node-link diagrams in which nodes are coloured and sized according to their values of change centrality. Since the change centrality is computed for each pair of subsequent time steps and is symmetrical, we chose to map its values in the visual features of the second time step of each pair; hence, the visual features of nodes for each time step express what has changed with reference to the previous one, while they are neutral for the first time step of the sequence. In this way, nodes that are present in only one time step of a pair are treated differently: new nodes (present only in the second time step of a pair) are highlighted, while dead nodes (present only in the first time step of a pair) are not. For highlighting dead nodes, we can change the perspective and map analytical values to the visual features of the first time step of each pair. In any case, because of our choice of the linear coefficients for the calculation of this metric, the new/dead nodes are visually prominent. At the same time, having chosen a logarithmic scale for the

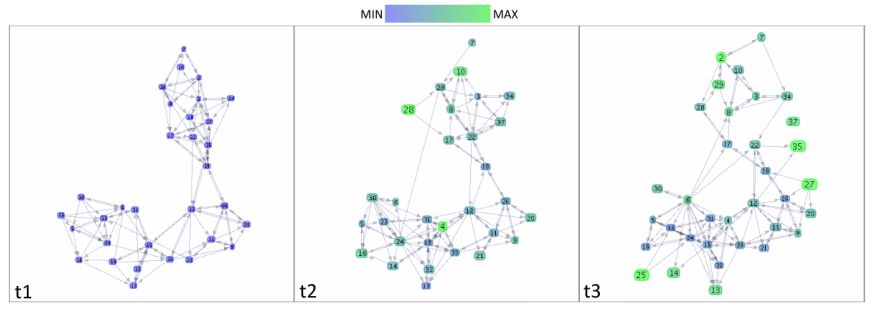

Fig. 2. Change centrality mapped to node size and colour in a juxtaposition view. Larger green nodes have encountered more changes in their connections from the previous time step. Smaller blue nodes are more stable. mapping of analytical measures to visual features, it is also possible to identify smaller differences due to changes in the far neighbours. On an overview level, we can observe that more nodes join the network at $t_{3}$ than at other time steps, but at $t_{3}$ there is also a group of nodes in the middle-bottom of the diagram that are less affected by change (and appear smaller and blueish).

Besides identifying appearing/disappearing nodes and comparing global changes between subsequent time steps, we might be interested to track the amount of change a given node encounters over time. To solve this task, we can use the $2.5 \mathrm{D}$ view and map the change centrality to both the colour and the thickness of node trajectories. In Figure 3, looking at the colour shading along the trajectory of a selected node, we see how its change centrality varies over time, being maximum at $t_{3}$ (green shade).

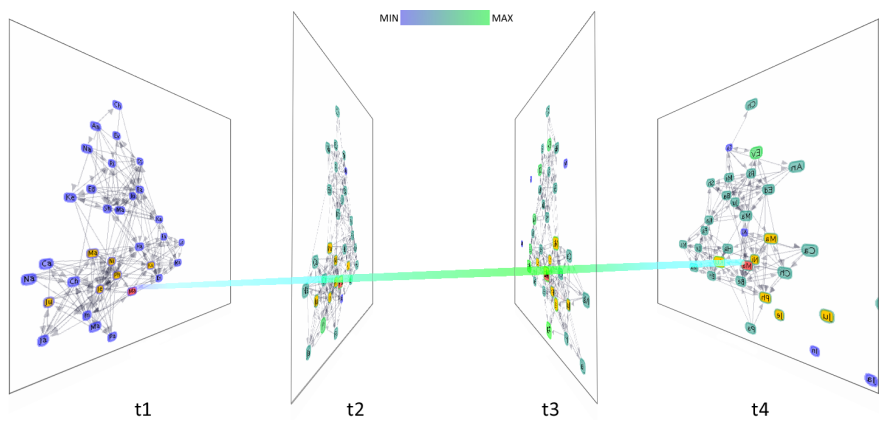

Fig. 3. Change centrality mapped to thickness and colour of a node trajectory in a $2.5 \mathrm{D}$ view. The selected node has encountered more changes in its links at time $t_{3}$ (trajectory is more green and thicker) than at other time steps.

\section{B. Dynamic layout}

The computation of a good layout is a crucial issue for the visualization of node-link diagrams, because of the perceptual prominence of position amongst all visual variables. In the case of dynamic networks the problem is even more demanding, since the layout has to change enough to reflect the evolution of the network, but not too much in order to not confuse the user. This problem, well known in the fields of graph drawing and visualization, related to enhancing the perception of changes while preserving the mental map [8]. Several methods have been proposed for solving this problem and Brandes at al. [22] group them in three main approaches: aggregation, anchoring, and linking. With aggregation, node positions are fixed, while in the latter cases, node positions change according to the network evolution, to a certain extent. Finding an optimal value for this extent is also an issue. Moreover, empirical studies have shown that the optimal amount of preservation of the mental map depends on the data and the task [23], [24].

In our previous work [19], we have adopted a linking approach combined with an interactive technique to enable the user to continuously control the stability of the layout. But this solution, as well as other methods based on linking 
and anchoring approaches, has the limitation that the default amount of displacement is the same for all nodes.

In this perspective, the change centrality metric can be useful to optimize the dynamic layout. After having computed the values of change centrality for the nodes of a network, we use them to tune the displacements between time steps in our dynamic layout.

All visualizations introduced in the figures above are obtained with this optimized dynamic layout, but its advantages are particularly clear if we consider a superimposition view (Figure 4). Looking at the length of the arrows composing node trajectories, we see the displacement of nodes across subsequent time steps. In the dynamic layout computed without the change centrality optimization, they all have the same length. In the one computed with the change centrality optimization, the length are different and reflect the actual relational changes of each node. In this way the layout provides not only a better overview of the network evolution, but also specific details of which nodes encountered larger changes and when.
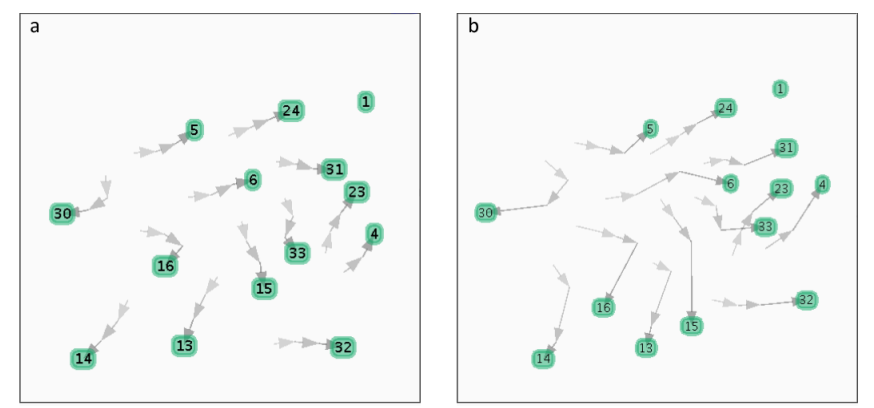

Fig. 4. Two superimposition views of the same network: a) dynamic layout without change centrality optimization - the extents of node displacements are all the same; b) dynamic layout with change centrality optimization - the extents of node displacements correspond to the amounts of change in their connections.

\section{CONCLUSION AND NEXT STEPS}

We have presented a set of novel metrics for the visual analysis of dynamic networks. Aiming to enhance the perception of change and the gaining of both overview and detailed insights on the network evolution, we have mapped these metrics to size and colour to enrich interactive visualizations based on node-link diagrams. Furthermore, we have shown how they can be taken into account for the computation of optimized dynamic layout. As future work, we plan to conduct user studies in order to evaluate the usability and the utility of these metrics to support different types of visual analytics tasks with different types of datasets.

\section{ACKNOWLEDGMENT}

Our research is funded by the Austrian Federal Ministry of Transport, Innovation and Technology (FFG 820928) and supported by the Laura Bassi Centre for Visual Analytics Science and Technology - CVAST (FFG 822746).

\section{REFERENCES}

[1] J. Ahn, C. Plaisant, and B. Shneiderman, "A task taxonomy of network evolution analysis," Tech Report HCIL-2011-09, Apr. 2011.

[2] J. Moody, D. McFarland, and S. BenderdeMoll, "Dynamic network visualization," Am. J. Sociol., vol. 110, no. 4, pp. 1206-1241, 2005.

[3] G. Robertson, R. Fernandez, D. Fisher, B. Lee, and J. Stasko, "Effectiveness of animation in trend visualization," TVCG, vol. 14, no. 6, pp. $1325-1332,2008$.

[4] L. Nowell, E. Hetzler, and T. Tanasse, "Change blindness in information visualization: A case study," in Proc. of the IEEE Symposium on Information Visualization (INFOVIS). Washington, DC, USA: IEEE Computer Society, 2001.

[5] J. Branke, "Dynamic graph drawing," in Drawing Graphs, ser. LNCS Springer, 2001, vol. 2025, pp. 228-246.

[6] M. Pohl, F. Reitz, and P. Birke, "As time goes by: integrated visualization and analysis of dynamic networks," in Proc. of the working conf. on Advanced visual interfaces (AVI). New York, NY, USA: ACM, 2008, pp. $372-375$.

[7] C. McGrath and J. Blythe, "Do you see what I want you to see ? the effects of motion and spatial layout on viewers perceptions of graph structure," JOSS, vol. 5, 2004.

[8] P. Eades and W. Lai, "Preserving the mental map of a diagram," in Proc. of the Int. Conf. on Computational Graphics and Visualization Techniques (COMPUGRAPHICS). Elsevier, 1991.

[9] S. Wasserman and K. Faust, Social Network Analysis: Methods and Applications. Cambridge University Press, 1994.

[10] C. C. Aggarwal, Social Network Data Analytics, 1st ed. Springer Publishing Company, Inc., 2011.

[11] S. Uddin and L. Hossain, "Time scale degree centrality: A time-variant approach to degree centrality measures," in Int. Conf. on Advances in Social Networks Analysis and Mining (ASONAM),, 2011, pp. 520 -524.

[12] K. Lerman, R. Ghosh, and J. H. Kang, "Centrality metric for dynamic networks," in Proc. of the Workshop on Mining and Learning with Graphs (MLG). New York, NY, USA: ACM, 2010, pp. 70-77.

[13] T. Y. Berger-Wolf and J. Saia, "A framework for analysis of dynamic social networks," in Proc. of the ACM SIGKDD int. conf. on Knowledge discovery and data mining (KDD). New York, NY, USA: ACM, 2006, pp. 523-528.

[14] D. Braha and Y. Bar-Yam, "From centrality to temporary fame: Dynamic centrality in complex networks," Complexity, vol. 12, no. 2, pp. 59-63, 2006.

[15] M. Pohl and S. Diehl, "What dynamic network metrics can tell us about developer roles," in Proc. of the int. workshop on Cooperative and human aspects of software engineering (CHASE). New York, NY, USA: ACM, 2008, pp. 81-84.

[16] M. Berlingerio, M. Coscia, F. Giannotti, A. Monreale, and D. Pedreschi, "Foundations of multidimensional network analysis," in Int. Conf. on Advances in Social Networks Analysis and Mining (ASONAM), 2011.

[17] P. Brodka, K. Skibicki, P. Kazienko, and K. Musial, "A degree centrality in multi-layered social network," in Int. Conf. on Computational Aspects of Social Networks (CASoN), 2011, pp. 237 -242.

[18] P. Jaccard, "The distribution of flora in the alpine zone," The New Phytologist, vol. 11, no. 2, pp. 37-50, 1912.

[19] P. Federico, W. Aigner, S. Miksch, F. Windhager, and L. Zenk, "A visual analytics approach to dynamic social networks," in Proc. of the Int. Conf. on Knowledge Management and Knowledge Technologies (i-KNOW). New York, NY, USA: ACM, 2011.

[20] D. Keim, F. Mansmann, J. Schneidewind, and H. Ziegler, "Challenges in visual data analysis," in Int. Conf. on Information Visualization, 2006.

[21] P. Federico, W. Aigner, S. Miksch, F. Windhager, and L. Zenk, "Visual analytics of dynamic networks - a case study," in The Int. UKVAC Workshop on Visual Analytics (VAW), 2011.

[22] U. Brandes, N. Indlekofer, and M. Mader, "Visualization methods for longitudinal social networks and stochastic actor-oriented modeling," Social Networks, to appear.

[23] H. C. Purchase, E. Hoggan, and C. Görg, "How important is the "mental map"?: an empirical investigation of a dynamic graph layout algorithm," in Proc. of the 14th int. conf. on Graph drawing (GD). Berlin, Heidelberg: Springer-Verlag, 2007, pp. 184-195.

[24] P. Saffrey and H. Purchase, "The "mental map" versus "static aesthetic" compromise in dynamic graphs: a user study," in Proc. of the conf. on Australasian user interface (AUIC). Darlinghurst, Australia: Australian Computer Society, Inc., 2008, pp. 85-93. 